TITLE:

\title{
Water-adsorption effect on electrical resistivity of nanoporous gold
}

$\operatorname{AUTHOR}(S)$ :

Hakamada, Masataka; Kato, Naoki; Miyazawa, Naoki; Mabuchi, Mamoru

\section{CITATION:}

Hakamada, Masataka ...[et al]. Water-adsorption effect on electrical resistivity of nanoporous gold. Scripta Materialia 2016, 123: 30-33

ISSUE DATE:

2016-10

URL:

http://hdl.handle.net/2433/237636

\section{RIGHT:}

(c) 2016. This manuscript version is made available under the CC-BY-NC-ND 4.0 license

http://creativecommons.org/licenses/by-nc-nd/4.0/; The full-text file will be made open to the public on 1 October 2016 in accordance with publisher's 'Terms and Conditions for Self-Archiving'.; この論文は出版社版でありません。引用の際 には出版社版をご確認ご利用ください。; This is not the published version. Please cite only the published version. 


\title{
Water-adsorption effect on electrical resistivity of nanoporous gold
}

\author{
Masataka Hakamada*, Naoki Kato ${ }^{1}$, Naoki Miyazawa and Mamoru Mabuchi \\ Department of Energy Science and Technology, Graduate School of Energy Science, Kyoto \\ University, Yoshidahonmachi, Sakyo, Kyoto, 060-8501, Japan
}

\begin{abstract}
The effect of water adsorption on the electrical resistivity of nanoporous gold (NPG) thin films, as well as flat gold (FG) with no nanoporosity, has been examined. Both NPG and FG thin films showed higher electrical resistivity in water than in air; however, the difference between the electrical resistivity of NPG in water and in air was quite large. The first-principles calculations suggest that the density of states at Fermi energy of the surface gold atoms decreases as a result of water adsorption, which is responsible for the electrical resistivity change in the NPG.
\end{abstract}

Keywords: Adsorption; Nanoporous gold; Electrical resistivity

* Corresponding author. E-mail address: hakamada.masataka.3x@kyoto-u.ac.jp (M. Hakamada).

1 Present address: Tanaka Kikinzoku Kogyo K. K., Japan. 
The adsorption of various molecules on the surface of a substrate can be detected by monitoring the electrical properties of the substrate material [1-5]. The substrate materials should possess nanostructures with both a high surface area and electrical conductivity. For example, adsorption of oxygen on carbon nanotubes [1,2], protein on silicon nanowires $[3,4]$ and thiol molecules on gold nanowires [5] can be successfully detected.

Nanoporous gold (NPG) has open porous structures with pores and ligaments in the nanometer size range and can be fabricated by dealloying or selective dissolution of less noble elements from binary alloys in an electrolyte [6,7]. Monolithic nanoporous metals with high specific surface areas exhibit adsorption-driven macroscopic actuation in aqueous and gaseous environments [8-14]. In particular, Detsi et al. have revealed the humidity dependence of a macroscopic strain in NPG [1214]; that is, adsorption of water in gaseous environments actuates NPG. The actuation of NPG by adsorption suggests that the electrical properties of NPG are also altered by the atmosphere. In addition, the conditions used for dealloying and post-treatment determine the pore and ligament sizes of NPG; under conditions that suppresses the self-organization of gold atoms, the pore and ligament sizes are as small as the mean free path of electrons in gold $[15,16]$. Consequently, the electrical properties of NPG are expected to be sensitive to the adsorption of water. Considering this background, we compare the change in electrical properties of NPG resulting from the adsorption of water. That is, the effects of the atmosphere (air and water) on the electric resistivity of NPG are examined. 
NPG films were fabricated by dealloying of Au0.25Ag0.75/pure-Au stacked film sputtered on an insulating glass substrate, as schematically shown in the Supplementary Material. First, a 70-nmthick gold adhesion layer was sputtered on the glass and then a 140-nm-thick Au0.25Ag 0.75 layer was sputtered on the gold adhesion layer by radio-frequency magnetron sputtering. A metallic mask was used for the fabrication of a circuit of sputtered film that is suitable for electrical resistivity measurements using a four-probe method. NPG films were produced by dealloying of the prepared film sample in 70 mass\% $\mathrm{HNO}_{3}$ for $24 \mathrm{~h}$ at $298 \mathrm{~K}$ (denoted as NPG-a), for $24 \mathrm{~h}$ at $253 \mathrm{~K}$ (denoted as NPG-b) and for 5 min at $298 \mathrm{~K}$ (denoted as NPG-c) under free corrosion conditions without anodic electrochemical potential. The dealloyed samples were thoroughly washed with distilled water to remove residual acid. For comparison, a 140-nm-thick flat gold (FG) film without a nanoporous structure was also prepared by sputtering on a glass substrate. The thickness of the sputtered films was confirmed using a probe-type step profiler (Dektak150 by ULVAC Corp.). The microstructure of the samples was observed by scanning electron microscopy (SEM).

The electrochemically active surface area of the gold films was evaluated by cyclic voltammetric (CV) measurements at room temperature using a potentiostat and a three-electrode electrochemical cell with Pt wire as a counter electrode, saturated calomel electrode (SCE) as a reference electrode, and sample film as a working electrode [17]. The electrolyte was $0.1 \mathrm{~mol} / \mathrm{L} \mathrm{H}_{2} \mathrm{SO}_{4}$ aqueous solution deoxygenated by bubbling with high purity $\mathrm{N}_{2}$ for 30 min prior to each experiment. CV was conducted at $0.5 \mathrm{mV} \mathrm{s}^{-1}$ in a potential range from 0 to $1.3 \mathrm{~V}$ vs. SCE. 
The electrical resistivity of the NPG and FG samples was measured at $298 \pm 0.5 \mathrm{~K}$ by a direct current four-probe method in air and in ion-exchanged and distilled water. The electrical resistivity of the NPG layers was calculated assuming that the two layers have a parallel connection for electron conductivity [18]. The change in the electrical resistivity $\left(\Delta \rho / \rho_{0}\right)$ of NPG and FG resulting from immersion in water was then calculated using the following equation:

$\Delta \rho / \rho_{0}=\left(\rho_{\mathrm{w}}-\rho_{0}\right) / \rho_{0}$,

where $\rho_{0}$ is the electrical resistivity of NPG or FG measured in air (with humidity of $30 \%$ ) and $\rho_{\mathrm{w}}$ is the electrical resistivity of NPG or FG measured in ion-exchanged and distilled water. The thermal equilibrium was confirmed by monitoring the electric potential during the measurements. To check reproducibility, three or four identical samples were fabricated and their electrical resistivity was measured.

The microstructures of the fabricated NPG and FG are shown in Fig. 1. Nanoporous structures were observed in NPG-a, b and c, whereas the SEM image of FG showed a featureless surface. The geometric characteristics of pore and ligament sizes in the NPG samples were clearly varied by the dealloying conditions. The roughness factor (RF, ratio of the electrochemically active surface area of NPG to that of FG) can be estimated by the following equation [17]:

$A_{\mathrm{NPG}} / A_{\mathrm{FG}}=Q_{\mathrm{NPG}} / Q_{\mathrm{FG}}$,

where $A$ is the surface area and $Q$ is the charge associated with the reduction of adsorbed oxygen, which can be calculated using the cathodic peak in the CV measurements. Table I summarizes the 
calculated roughness factors as well as the pore and ligament sizes, which were measured from the SEM images of the NPG samples. When the pore and ligament sizes of NPG are comparable, the specific surface area $(S)$ can be calculated by

$S=C / \rho d_{L}$

where $\rho$ is the density of solid bulk gold $\left(=19.3 \mathrm{~g} / \mathrm{m}^{3}\right), d_{\mathrm{L}}$ is the ligament size of NPG and $C$ is a constant (= 3.7) [19]. In the present study, $S$ of NPG-b with equivalent pore and ligament sizes was calculated and then $S$ of NPG-a and NPG-c with inequivalent pore and ligament sizes are calibrated by the roughness factor. As a result, $S$ roughly increased as the pore size decreased, assuming that oxygen monoatomically adsorbs (and desorbs) on NPG in the CV measurements, as shown in Table 1.

The measured electrical resistivity of FG was $37.6 \mathrm{n} \Omega \mathrm{m}$, which is slightly higher than that reported in the literature [20]. This indicates that the electrical resistivity of FG is dominated by free electron scattering at the surface because the thickness of the FG film is in the same length order of the mean free path of the electrons [15,16]. The electrical resistivity of NPG in air at 298 K was 246 (NPG-a), 426 (NPG-b), and 548 (NPG-c) $n \Omega$ m. The measured resistivity of the present NPG was in agreement with other literature data (shown in Supplementary Material) [21-25], which indicates the validity of the present measurement.

The change in the electrical resistivity of NPG and FG after immersion in water $\left(\Delta \rho / \rho_{0}\right)$ are shown as a function of specific surface area in Fig. 2. The $\Delta \rho / \rho_{0}$ of the NPG samples was above $4 \%$, whereas 
that of FG was $0.8 \%$. The $\Delta \rho / \rho_{0}$ of NPG increased with specific surface area, suggesting that the adsorption of water on the surface of the gold ligaments clearly affects the electrical properties of NPG. However, $\Delta \rho / \rho_{0}$ is not simply proportional to specific surface area. The $\Delta \rho / \rho_{0}$ of NPG-b and NPG-c was lower than that predicted by the extrapolation of data from FG and NPG-a; that is, when the pore size of NPG is smaller than a certain threshold, the smallness of the ligaments and pore sizes has a reverse effect on the electrical resistivity.

Dealloyed nanoporous metals have lattice distortion at the surface owing to a large curvature of the ligaments [26,27]. The coexistence of lattice expansion (positive strain) and compression (negative strain) owing to the peculiar nanostructure is one of the characteristics [26]. The surface adsorption and lattice distortion may synergistically affect the electrical conductivity of gold. In addition, adsorption effects have been investigated by density of states (DOS) of a metal atom at Fermi energy (FE) by electron state calculations [28,29]. Therefore, using first-principles calculations, water molecules are set on a gold (111) surface with or without lattice strain change of -5 to $+5 \%$, and DOS at FE as well as the adsorption energy of water molecules are calculated.

First, a Au (111) surface model with a surface unit cell of $(4 \times 4)$ was prepared. The simulation cell consisted of seven (111) gold layers and contained 112 gold atoms, which is called the $0 \%$ model in the present paper. A vacuum gap of $15 \AA$ was added to create the surface. Lattice strains from -5 to $+5 \%$ into the (111) layers of the gold surface model were loaded as NPG surface models, where the 
lattice spacings of $(01-1)$ and (-110) were simultaneously altered. The geometry optimization calculations using first-principles calculations were performed on each model.

The first-principles calculations were performed using Cambridge Serial Total Energy Package (CASTEP) [30], whereby a plane-wave basis set is used to calculate the electronic properties based on density functional theory (DFT) [31,32]. The Perdew—Burke and Ernzerhof version [33] of the generalized gradient approximation was used to represent exchange-correlation functionals in the DFT. Ultrasoft pseudopotentials [34] were used for all elements in the calculations. The cutoff energy was set to $600 \mathrm{eV}$ with a Gaussian smearing of $0.1 \mathrm{eV}$ in width in all calculations. The Brillouin zone of $4 \times 4 \times 1$ was sampled using Monkhorst--Pack k-point mesh [35]. The gold atoms in the bottom two layers were fixed during geometry optimizations.

As shown in Fig. 3a, four water molecules were placed on the top sites of the (111) surface of gold in accordance with the previous study [36]. The adsorption energy ( $\left.E_{\text {ads }}\right)$ of a molecule is evaluated as:

$E_{\mathrm{ads}}=\left(E_{\mathrm{tot}}-E_{\mathrm{Au}}-n E_{\mathrm{water}}\right) / n$,

where $E_{\text {tot }}$ is the energy of the total system, $E_{\text {Au }}$ is the energy of the gold (111) surface model without adsorption of water, $E_{\text {water }}$ is the energy of one water molecule and $n$ is the number of adsorbed water molecules (=4). For reference, the (111) surface of gold without any adsorbents was also modeled and calculated. 
The relationship between $E_{\text {ads }}$ and lattice strain in gold is shown in Fig. 3 b. Eads varies monotonically with lattice strain. That is, the positive lattice strain (=lattice expansion) leads to a more stable adsorption of water on the gold (111) surface and vice versa. Conversely, the DOS at FE of the gold atom on which the water molecule adsorbs was significantly lowered by water adsorption, irrespective of the lattice strain, as shown in Fig. 3c. The behavior of the conductive electron in a metal depends much a lot on the electrons at the Fermi surface and is proportional to DOS at FE [3739]; hence, the increase in the electrical resistivity of NPG, which is the reciprocal of electrical conductivity, can be attributed to the lowering of DOS at FE of the gold atoms, possibly owing to the polar nature of the water molecule.

Fig. $3 \mathrm{c}$ reveals that when a lattice strain of $-5,-2.5$ and $+5 \%$ is introduced in the water-adsorbed gold (111) surface, the DOS at FE increases compared with no lattice strain. This suggests that a large surface lattice strain promotes electrical conductivity at the surface layer of the gold surface. Smaller pore and ligament sizes in NPG cause a larger lattice strain at the surface of the ligaments owing to a large curvature; therefore, the lower gradient at larger specific surface area in Fig. 2, that is, the breakdown of the dependence of $\Delta \rho / \rho 0$ on the surface area of NPG, may be partly attributed to a large lattice strain owing to a small ligament size, although there is much room for study.

In conclusion, the difference between the electrical resistivity of NPG samples in air and in water $\left(\Delta \rho / \rho_{0}\right)$ was evaluated and shown to be more than four times that of FG. The $\Delta \rho / \rho_{0}$ of NPG roughly increased as the pore size was reduced and consequently the specific surface area increased; however, 
$\Delta \rho / \rho_{0}$ was not simply proportional to the surface area; that is, the smaller pore size suppressed the increase in $\Delta \rho / \rho_{0}$, in spite of the large surface area. The first-principles calculations indicate that DOS at FE of the surface gold atom decreases after water adsorption and increases from lattice strain. Hence, the electrical properties, or electron conducting behavior, in NPG strongly depends on water adsorbed on the surface.

The author M. M. thanks the Japan Prize Foundation for the financial support for experiments. Computation time was provided by the SuperComputer System, Institute for Chemical Research, Kyoto University, which the authors acknowledge. Observations by SEM were conducted in Research and Education Center of Advanced Energy Science, Graduate School of Energy Science, Kyoto University.

\section{Appendix A. Supplementary material}

Supplementary material to this article can be found online at [URL will be inserted by Elsevier] for a schematic diagram and details of the fabrication procedure and for comparison of the electrical resistivity with literature data.

\section{References}

[1] P.G. Collins, K. Bradley, M. Ishigami, A. Zettl, Science 287 (2000) 1801-1804.

[2] M. Shim, A. Javey, N.W.S. Kam, H. Dai, J. Am. Chem. Soc. 123 (2001) 11512-11513. 
[3] G. Zheng, F. Patolsky, Y. Cui, W.U. Wang, C.M. Lieber, Nature Biotechnol. 23 (2005) 12941301.

[4] Y. Cui, Q. Wei, H. Park, C.M. Lieber, Science 293 (2001) 1289-1292.

[5] A. Kisner, M. Heggen, D. Mayer, U. Simon, A. Offenhäusser, Y. Mourzina, Nanoscale 6 (2014) 5146-5155.

[6] A. J. Forty, Nature 282 (1979) 597-598.

[7] J. Erlebacher, M.J. Aziz, A. Karma, N. Dimitrov, K. Sieradzki, Nature 410 (2001) 450-453.

[8] D. Kramer, R.N. Viswanath, J. Weissmüller, Nano Lett. 4 (2004) 793-796.

[9] J. Biener, A. Wittstock, L.A. Zepeda-Ruiz, M.M. Biener, V. Zielasek, D. Kramer, R.N. Viswanath, J. Weissmüller, M. Bäumer, A.V. Hamza, Nat. Mater. 8, (2009) 47-51.

[10] H.-J. Jin, X.-L. Wang, S. Parida, K. Wang, M. Seo, J. Weissmüller, Nano Lett. 10 (2010) 187194.

[11] M. Hakamada, S. Matsumura, M. Mabuchi, Mater. Lett. 70 (2012)132-134.

[12] E. Detsi, Z.G. Chen, W.P. Vellinga, P.R. Onck, J.T.M. De Hosson, Appl. Phys. Lett. 99 (2011) 083104.

[13] E. Detsi, Z.G. Chen, W.P. Vellinga, P.R. Onck, J.T.M. De Hosson, J. Nanosci. Nanotechnol. 12 (2012) 4951-4955.

[14] E. Detsi, S.H. Tolbert, S. Punzhin, J.T.M. De Hosson, J. Mater. Sci. 51 (2016) 615-634.

[15] E.H. Sondheimer, Adv. Phys. 1 (1952) 1-42. 
[16] K. Fuchs, Proc. Cambridge Philos. Soc. 34 (1938) 100-108.

[17] S. Trasatti, O.A. Petrii, J. Electroanal. Chem. 327 (1992) 353-376.

[18] K. Nakamura, S. Kikuchi, J. Jpn. Inst. Met. Mater. 72 (2008) 800-803 (in Japanese).

[19] E. Detsi, E. De Jong, A. Zinchenko, Z. Vuković, I. Vuković, S. Punzhin, K. Loos, G. ten Brinke, H.A. De Raedt, P.R. Onck, J.T.M. De Hosson, Acta Mater. 59 (2011) 7488-7497.

[20] D.A. Butts, D. Taarea, Electrical Properties, in: W.F. Gale, T.C. Totemeier (Eds.), Smithells Metals Reference Book, Eighth Edition, Elsevier, Oxford, 2004, p. 19-1.

[21] R.W. Ertenberg, B. Andraka, Y. Takano, Physica B 284-288 (2000) 2022-2023.

[22] T. Fujita, H. Okada, K. Koyama, K. Watanabe, S. Maekawa, M.W. Chen, Phys. Rev. Lett. 101 (2008) 166601.

[23] E. Seker, M. Reed, M. Utz, M.R. Begley, Appl. Phys. Lett. 92 (2008) 154101.

[24] R. Xia, J.L. Wang, R. Wang, X. Li, X. Zhang, X.Q. Feng, Y. Ding, Nanotechnology 21 (2010) 085703.

[25] Tanimoto, T. Shintani, H. Mizubayashi, Probable Carrier Localization in Nanoporous Gold Prepared by Selective Etching, in: B.-Y. Hur, B.-K. Kim, S.-E. Kim, S.-K. Hyun (Eds.), Porous Metals and Metallic Foams-MetFoam 2011, GS-intervision, Seoul, 2012, pp. 655-660.

[26] M. Hakamada, H. Nakano, T. Furukawa, M. Takahashi, M. Mabuchi, J. Phys. Chem. C 114 (2010) 868-873. 
[27] S. Parida, D. Kramer, C.A. Volkert, H. Rösner, J. Erlebacher, J. Weissmüller, Phys. Rev. Lett. 97 (2006) 035504.

[28] P. Jelínek, M. Švec, P. Pou, R. Perez, V. Cháb, Phys. Rev. B 77 (2008) 115447.

[29] A. Roy, T. Pandey, N. Ravishankar, A.K. Singh, J. Phys. Chem. C 118 (2014) 18676-18682.

[30] M.C. Payne, M.P. Teter, D.C. Allan, T.A. Arias, J.D. Joannopoulos, Rev. Mod. Phys. 64, (1992) 1045-1097.

[31] P. Hohenberg, W. Kohn, Phys. Rev. B 136 (1964) 864-871.

[32] W. Kohn, L. Sham, Phys. Rev. A 140 (1965) 1133-1138.

[33] J.P. Perdew, K. Burke, M. Ernzerhof, Phys. Rev. Lett. 77 (1996) 3865-3868.

[34] D. Vanderbilt, Phys. Rev. B 14 (1990) 7892-7895.

[35] H.J. Monkhorst, J.D. Pack, Phys. Rev. B 13 (1976) 5188-5192.

[36] R. Nadler, J.F. Sanz, J. Chem. Phys. 137 (2012) 114709.

[37] J.M. Ziman, Principles of the Theory of Solids,Cambridge University Press, Cambridge, 1964, p. 215.

[38] N.D. Lang, Ph. Avouris, Phys. Rev. B 62 (2000) 7325-7329.

[39] K. Yokota, M. Taniguchi, T. Kawai, J. Phys. Chem. C 114 (2010) 4044-4050. 


\section{Tables}

Table 1. Ligament sizes, pore sizes, roughness factors and calculated specific surface areas of NPG samples.

\begin{tabular}{lcccc}
\hline \hline \multirow{2}{*}{ Sample ID } & $\begin{array}{c}\text { Ligament diameter, } \\
\text { Pore diameter, }\end{array}$ & Roughness factor, & Specific surface area, \\
& $d_{\mathrm{L}}(\mathrm{nm})$ & $d_{\mathrm{p}}(\mathrm{nm})$ & $A_{\mathrm{NPG}} / A_{\mathrm{FG}}$ & $S\left(\mathrm{~m}^{2} / \mathrm{g}\right)$ \\
\hline NPG-a & 77 & 53 & 16 & 3.4 \\
NPG-b & 24 & 24 & 38 & 8.0 \\
NPG-C & 23 & 14 & 54 & 11.3 \\
\hline \hline
\end{tabular}




\section{Figure captions}

Fig. 1. SEM images of nanoporous gold (NPG-a, NPG-b and NPG-c) (a-c) and flat gold (FG) (d).

Fig. 2. Relationship between the change in electrical resistivity after immersion in water and the specific surface area of nanoporous gold (NPG) and flat gold (FG) at 298 K. Error bars are standard deviations.

Fig. 3. (a) Top (upper) and side (lower) views of the atomic model of water on the gold (111) surface. Yellow, red and white spheres are gold, oxygen and hydrogen atoms, respectively. Four water molecules are located on the top site of the gold (111) surface. (b) Relationship between the lattice strain in gold and the adsorption energy of water. (c) Relationship between lattice strain in gold and density of states of surface gold atom. 

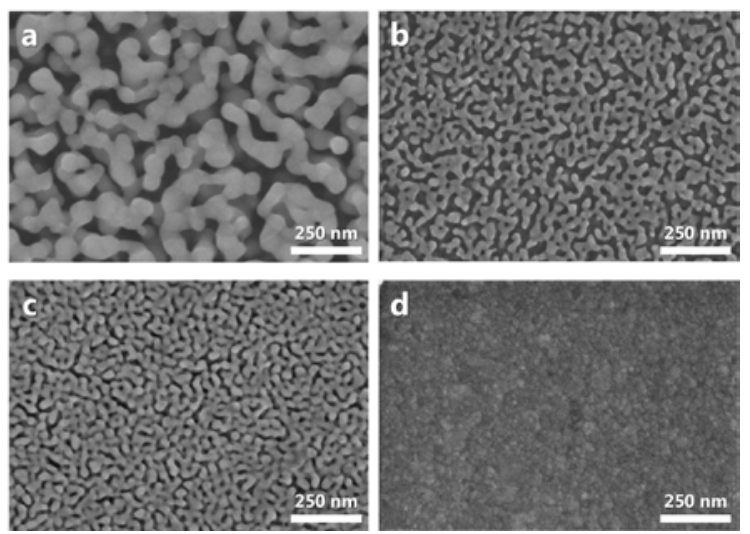

Fig. 1. SEM images of nanoporous gold (NPG-a, NPG-b and NPG-c) (a-c) and flat gold (FG) (d). 


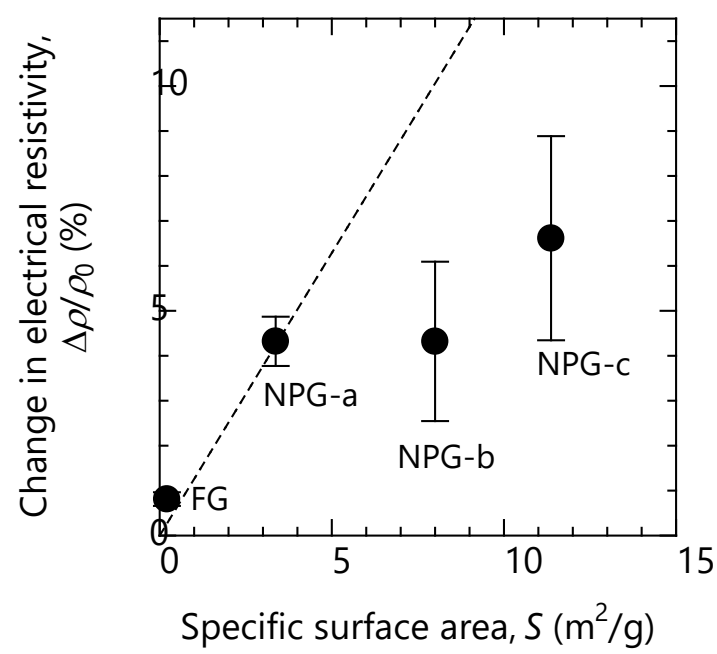

Fig. 2. Relationship between the change in electrical resistivity after immersion in water and the specific surface area of nanoporous gold (NPG) and flat gold (FG) at $298 \mathrm{~K}$. Error bars are standard deviations. 


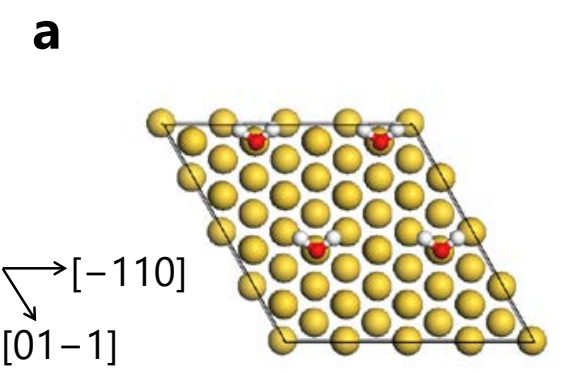

b
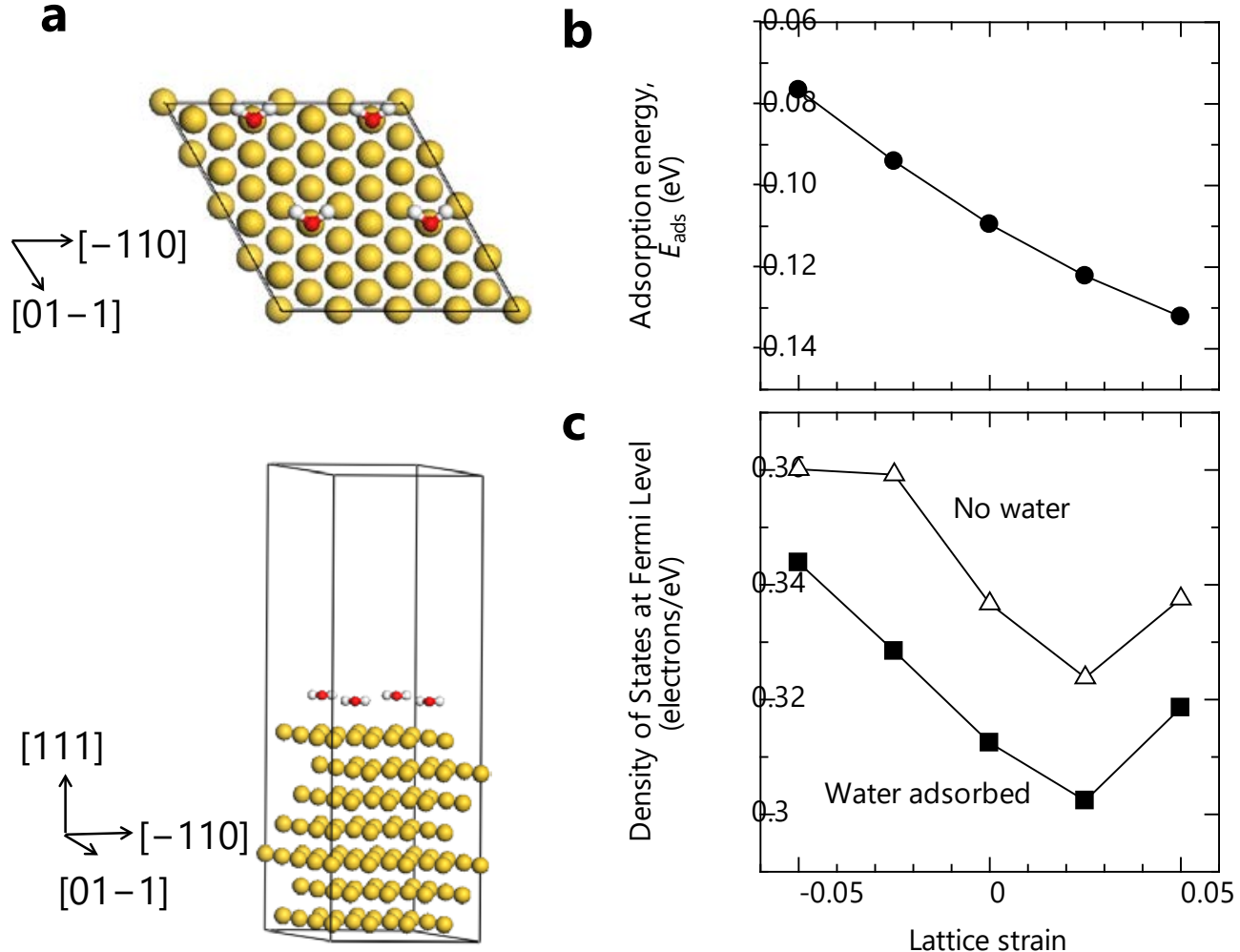

C

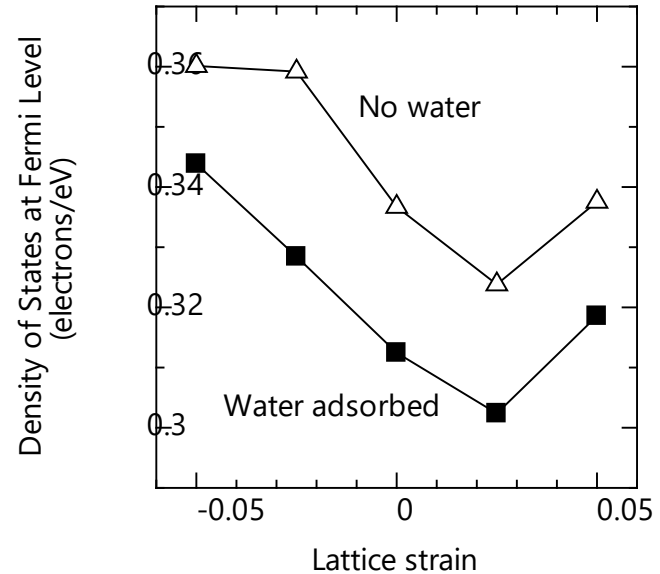

Fig. 3. (a) Top (upper) and side (lower) views of the atomic model of water on the gold (111) surface. Yellow, red and white spheres are gold, oxygen and hydrogen atoms, respectively. Four water molecules are located on the top site of the gold (111) surface. (b) Relationship between the lattice strain in gold and the adsorption energy of water. (c) Relationship between lattice strain in gold and density of states of surface gold atom. 


\title{
Supplementary Material for:
}

\section{Water-adsorption effect on electrical resistivity of nanoporous gold}

\author{
Masataka Hakamada*, Naoki Kato ${ }^{1}$, Naoki Miyazawa and Mamoru Mabuchi \\ Department of Energy Science and Technology, Graduate School of Energy Science, Kyoto \\ University, Yoshidahonmachi, Sakyo, Kyoto, 060-8501, Japan
}

* Corresponding author. E-mail address: hakamada.masataka.3x@kyoto-u.ac.jp (M. Hakamada).

1 Present address: Tanaka Kikinzoku Kogyo K. K., Japan.

\section{Fabrication of nanoporous gold for measurement of electrical resistivity}

Nanoporous gold (NPG) films were fabricated by dealloying of Au0.25Ag0.75/pure-Au stacked film sputtered on an insulating glass substrate, as schematically shown in Fig. S1. First, a 70-nm-thick gold adhesion layer was sputtered on the glass and then a 140-nm-thick Au0.25Ag0.75 layer was sputtered on the gold adhesion layer by radio-frequency magnetron sputtering. A metallic mask was used for the fabrication of a circuit of sputtered film that is suitable for electrical resistivity measurements using a four-probe method.

(a)

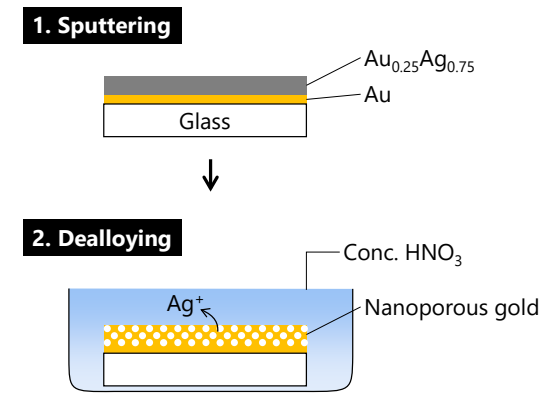

(b)

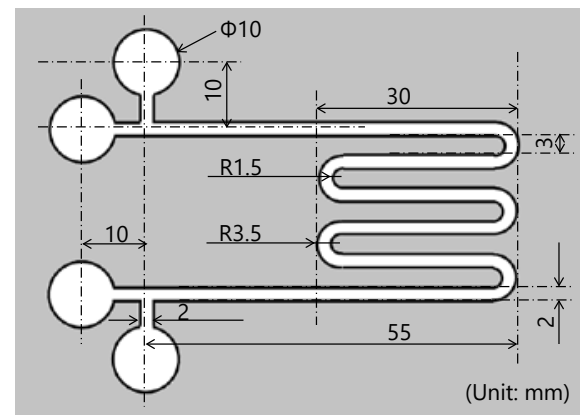

Fig. S1. (a) Schematic illustration of the fabrication of nanoporous gold thin film for measurement of electrical resistivity. (b) Metallic mask used for sputtering. 


\section{Comparison of the electrical resistivity of nanoporous gold with literature data}

The electrical resistivity of NPG in air at $298 \mathrm{~K}$ was in agreement with the other literature data [1-5], as summarized in Fig. S2, which indicates the validity of the present measurement.

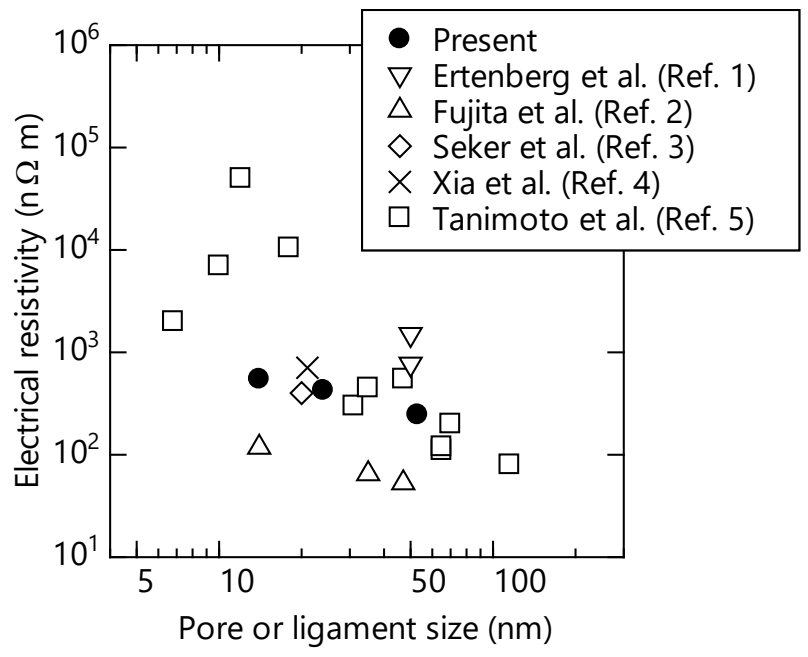

Fig. S2. Comparison of the electrical resistivity of nanoporous gold in the present study with other literature data.

\section{References}

[1] R.W. Ertenberg, B. Andraka, Y. Takano, Physica B 284-288 (2000) 2022.

[2] T. Fujita, H. Okada, K. Koyama, K. Watanabe, S. Maekawa, M.W. Chen, Phys. Rev. Lett. 101, (2008) 166601.

[3] E. Seker, M. Reed, M. Utz, M. R. Begley, Appl. Phys. Lett. 92 (2008) 154101.

[4] R. Xia, J. L. Wang, R. Wang, X. Li, X. Zhang, X.Q. Feng, Y. Ding, Nanotechnology 21 (2010) 085703.

[5] H. Tanimoto, T. Shintani, H. Mizubayashi, Probable Carrier Localization in Nanoporous Gold Prepared by Selective Etching, in: B.-Y. Hur, B.-K. Kim, S.-E. Kim, S.-K. Hyun (Eds.), Porous Metals and Metallic Foams-MetFoam 2011, GS-intervision, Seoul, 2012, pp. 655-660. 